\title{
Dynamic Influences in Multi-Component Maintenance
}

\author{
Ralph E. Wildeman and Rommert Dekker \\ Erasmus University Rotterdam
}

September 11, 1996

\begin{abstract}
Most maintenance-optimisation models assume an infinite planning horizon and suppose that the failure process is stationary. Hence, information which is not known beforehand and which becomes available on the short term only, must be ignored. We consider in this paper a multi-component system with economically dependent components, and we compare the costs of a stationary-planning method with the costs according to an approach which can adapt this long-term plan to dynamically changing information (such as a variable use of components and the occurrence of maintenance opportunities). With numerical experiments we show that incorporating short-term information can yield considerable cost savings.
\end{abstract}

Keywords: Maintenance, multiple components, planning, opportunities, dynamic influences.

\section{Introduction}

When we develop a maintenance-optimisation model, we usually consider an infinite planning horizon and assume a long-term stable situation. This stationarity assumption facilitates the mathematical analysis and enables us to determine for example long-term maintenance frequencies, or control limits for carrying out maintenance depending on the state of the system. However, such a long-term view prevents incorporating information that becomes available on the short term only. Adapting the long-term plan according to short-term information may yield considerable cost savings.

Since many technical systems consist of multiple components, we will not consider maintenance of single components here, but we will take interactions between the components into account. Interactions between components can be classified into different types (see e.g. Thomas 1986), but here we will restrict ourselves to so-called economic dependence, where savings can be obtained when maintenance activities on different components are jointly carried out. As an example of a system with economically dependent components, consider an offshore installation with several machines, in which the maintenance of each component (a machine) requires preparatory or set-up work (say the transportation of a maintenance crew by air), which can be shared when several components are maintained simultaneously. The cost of this set-up work is called the set-up cost and may also consist of the down-time cost due to production loss if the system cannot be used during maintenance. Other examples of systems with economically dependent components are a transportation fleet consisting of multiple vehicles, or a road divided into road segments.

The relevance of incorporating short-term information, especially for multi-component maintenance, follows from a number of observations. For example, a maintenance rule for a single component can be formulated in several time scales or use indicators, such as calendar time, 
the number of running hours, or (in case of airplanes) the number of take-offs and landings. However, when maintenance of multiple components is to be planned and coordinated, the rules must be characterised by the same use indicator, and hence we have to consider calendar time. This implies that we have to assume an average utilisation factor for each component. Since the actual utilisation may fluctuate, and since this fluctuation is not known on the long term, the incorporation of short-term information indicating the real use, may improve the efficiency of the planning.

A similar argument holds for other short-term information, such as the occurrence of maintenance opportunities. Usually it is not known beforehand when such opportunities occur. It is of course possible to assume an average occurrence and to incorporate this in the long-term planning; however, the real occurrence is simply unknown and hence the planning may be improved when these unexpected events can be exploited once they happen.

Other short-term circumstances are for example fluctuations in costs, or a changing deterioration process due to varying weather conditions.

Considering dynamically changing information is only important if the variability in (for example) the components' use is that high, or the occurrence of maintenance opportunities is that frequent, that ignoring this information (and hence following a long-term plan under all circumstances) leads to unnecessarily high costs. Indeed, if components are used on a regular basis and opportunities rarely appear, that is, if the practical situation does not deviate much from a stationary situation, then following a long-term plan will not be more expensive than adapting this plan to the short-term.

In the literature of multi-component maintenance models with economic dependence, many methods are now available for generating a long-term (i.e., stationary) maintenance plan. For a recent overview we refer the reader to the review article of Dekker, Van der Duyn Schouten and Wildeman (1996). However, hardly any methods exist that deal with dynamically changing information. (This does not only hold for maintenance of multi-component systems, but for maintenance models in general.) Dekker, Van der Duyn Schouten and Wildeman report only one efficient approach for short-term planning, viz. the rolling-horizon approach by Wildeman, Dekker and Smit (1996).

In this paper we show how the short-term approach of Wildeman, Dekker and Smit (1996) can be extended to adapt a long-term plan to short-term circumstances. For the construction of a long-term plan, we apply the stationary-planning approach of Wildeman et al. (1995). Furthermore, we show that the dynamic approach is consistent with the stationary approach, i.e., that the approaches generate the same strategies in a stationary situation. Finally, to obtain insight in the effect of operational (i.e., short-term) circumstances, we consider in this paper the influence of two short-term aspects, viz. a variable use of components and the occurrence of maintenance opportunities. We investigate how these dynamic factors influence the costs of the maintenance planning.

This paper is organised as follows. In Section 2 we discuss the stationary-planning approach of Wildeman et al. (1995) and the dynamic-planning approach of Wildeman, Dekker and Smit (1996). We show how the long-term approach can be used as a basis for the dynamic approach, and that the approaches are consistent. We proceed in Section 3 by considering the effect of the components' variable use on the costs. We show how much can be saved by dynamic planning compared to stationary planning, given the variability in the utilisation. Similarly, we investigate the influence of opportunities in Section 4. In Section 5 we draw conclusions. 


\section{Description of Stationary- and Dynamic-Planning Approach}

In the following, we consider a multi-component system with components $i, i=1, \ldots, n$. Creating an occasion for preventive maintenance on one or more of these components involves a set-up cost $S$, independent of how many components are maintained. Because of this set-up cost $S$ there is an economic dependence between the individual components.

On an occasion for maintenance, component $i$ can be preventively maintained at an extra cost of $s_{i}$. Let $M_{i}(x)$ denote the expected cumulative deterioration costs of component $i$ (due to failures, repairs, operating costs, etc.), $x$ time units after its latest preventive maintenance. We assume that $M_{i}(\cdot)$ is strictly convex and that after preventive maintenance a component can be considered as good as new.

Denote now by $\Phi_{i}(x)$ the average costs of component $i$ over an infinite horizon, when component $i$ is preventively maintained on an occasion every $x$ time units. It is easy to obtain the following expression for $\Phi_{i}(x)$ (see, for example, Dekker 1995):

$$
\Phi_{i}(x)=\frac{s_{i}+M_{i}(x)}{x}, x>0 .
$$

With these preliminaries, we are now ready to discuss a stationary- and a dynamic-planning approach for the above multi-component system.

\subsection{Stationary Planning}

Wildeman et al. (1995) apply the following strategy for determining coordinated maintenance frequencies for the components $i, i=1, \ldots, n$.

They assume that every $T$ time units an occasion for preventive maintenance is created, and that component $i$ is preventively maintained at the integer multiple $k_{i} T$ of $T$. For example, let $T$ be equal to one month, and $k_{1}=1$ and $k_{2}=3$, then component 1 is preventively maintained every month, and component 2 every three months.

This strategy originates from inventory theory (see Goyal 1973) and was introduced in maintenance by Goyal and Kusy (1985) and further developed by Goyal and Gunasekaran (1992). The strategy is called indirect grouping (see Van Eijs, Heuts and Kleijnen 1992), since the groups are not fixed over time, but are formed indirectly when the maintenance of different components coincides. An alternative approach is direct grouping, where the components are partitioned into a number of fixed groups and are then always maintained in these groups. The advantage of indirect grouping compared to direct grouping, is that indirect grouping performs better and is easier to solve. For more details, see Dekker, Van der Duyn Schouten and Wildeman (1996).

Under the indirect-grouping strategy, the total average costs are equal to the average set-up cost and the sum of the individual average-cost functions $\Phi_{i}(\cdot)$. Hence, we have the following problem:

$$
\inf \left\{\frac{S}{T}+\sum_{i=1}^{n} \Phi_{i}\left(k_{i} T\right): k_{i} \in \mathbb{N}, T>0\right\} .
$$

This problem is a mixed continuous-integer programming problem, and in general such problems are difficult to solve. Goyal and Kusy (1985) and Goyal and Gunasekaran (1992) apply an easy iterative heuristic for determining values for $T$ and $k_{i}$. However, there approach has two main drawbacks. The first is that it works well only for simple (viz. polynomial) functions $M_{i}(\cdot)$, and 
furthermore that it is not optimal and that there is no information of how good the generated solutions are.

Wildeman et al. (1995) show that when the functions $M_{i}(\cdot)$ are convex, optimally solving the problem is relatively easy. First, the authors solve a relaxed problem, in which the constraints $k_{i} \in \mathbb{N}$ are replaced by $k_{i} \geq 1$. The solution of this relaxed problem is subsequently used in a fast solution approach for problem (2). If all $M_{i}(\cdot)$ are convex, a solution can be found with an arbitrarily small deviation from the optimal value in very little (almost linear) time by using Lipschitz optimisation. The authors show that for several well-known maintenance models, such as the minimal-repair model and the inspection model, the functions $M_{i}(\cdot)$ are convex. For other cases, the authors present heuristics that perform better than previously published ones.

\section{Example}

Consider a system with eight components that are maintained according to a standard minimalrepair model (see e.g. Dekker 1995). This implies that component $i$ is preventively replaced at fixed intervals of length $x$, with failure repair occurring whenever necessary; a failure repair restores the component into a state as good as before. Consequently, the deterioration costs are given by $M_{i}(x)=c_{i}^{r} \int_{0}^{x} r_{i}(t) d t$, with $r_{i}(\cdot)$ denoting the rate of occurrence of failures, and $c_{i}^{r}$ the failure-repair cost. Here $M_{i}(x)$ expresses the expected repair costs incurred in the interval $[0, x]$ due to failures. We assume that failures occur according to a Weibull process with scale parameter $\lambda_{i}>0$ (in weeks), and shape parameter $\beta_{i}>1$, which implies that $r_{i}(\cdot)$ is given by

$$
r_{i}(x)=\frac{\beta_{i}}{\lambda_{i}}\left(\frac{x}{\lambda_{i}}\right)^{\beta_{i}-1}
$$

We choose a Weibull process since the Weibull distribution has the most frequent application to fitting lifetime distributions (see e.g. O'Connor 1985).

Notice that by (3), we obtain the following expression for the function $M_{i}(\cdot)$ :

$$
M_{i}(x)=c_{i}^{r}\left(\frac{x}{\lambda_{i}}\right)^{\beta_{i}} .
$$

Since this function is (strictly) convex (for $\beta_{i} \geq 1$ ), we can apply the approach of Wildeman et al. (1995) to obtain an solution of problem (2) with an arbitrarily small deviation from the optimal solution.

As an example, suppose that the data for the eight components are given by Table I, and assume that the set-up cost $S=100$.

Table I

Example Data for Eight Components

\begin{tabular}{rrrrrrrrr}
\hline$i$ & 1 & 2 & 3 & 4 & 5 & 6 & 7 & 8 \\
\hline$\lambda_{i}$ & 8 & 7 & 9 & 14 & 6 & 15 & 3 & 5 \\
$\beta_{i}$ & 1.70 & 1.70 & 2.00 & 2.00 & 1.70 & 2.00 & 1.25 & 1.75 \\
$s_{i}$ & 105 & 225 & 345 & 165 & 500 & 345 & 105 & 345 \\
$c_{i}^{r}$ & 92 & 182 & 28 & 30 & 172 & 30 & 90 & 50 \\
\hline
\end{tabular}


Solving problem (2) for these data, using the approach of Wildeman et al. (1995) with a relative precision of $0.001 \%$, we obtain the following solution: $T=11.6,\left(k_{1}, \ldots, k_{8}\right)=$ $(1,1,3,3,1,4,1,2)$, with corresponding average costs equal to 321 .

Hence, every 11.6 weeks an occasion for preventive maintenance is created. Components 1, 2, 5 and 7 are maintained every occasion (i.e., every 11.6 weeks), component 8 is maintained every two occasions (every 23.2 weeks), components 3 and 4 are maintained every three occasions (every 34.8 weeks), and finally, component 6 is maintained every four occasions (every 46.4 weeks). (We do not round here - which will usually be done in practice- since that may disturb the comparison.)

\subsection{Dynamic Planning}

With the approach of the previous section, we can coordinate the frequencies of the multiple components in the system. However, we have assumed stationarity and we did not take shortterm circumstances into account. What happens if the utilisation of components is not constant but changes over time? Or how should opportunities be incorporated?

Below we will propose a rolling-horizon approach in which the long-term plan generated by the approach described in Section 2.1 can be adapted to deal with short-term information. The approach is an extension of the approach of Wildeman, Dekker and Smit (1996) and consists of five phases.

\section{Phase 1: Decomposition}

Apply a decomposition by determining in some way for each component an individual infinitehorizon maintenance rule. These individual rules may or may not take the economic dependence between components into account.

In this paper we apply the approach discussed in Section 2.1 to obtain individual (but coordinated) maintenance rules for the components. Hence, component $i$ is maintained every $x_{i}^{*}:=k_{i} T$ time units, where $T$ and $\left(k_{1}, \ldots, k_{n}\right)$ is an optimal solution of the indirect-grouping problem (2).

The important notion in this phase is the decomposition, where each component is considered separately (though its maintenance rule may be coordinated with other rules, as is done here). Usually, this phase has to be carried out only once.

\section{Phase 2: Penalty Functions}

We then derive a penalty function $h_{i}(\Delta t)$ for each component $i$, expressing the expected costs of shifting the preventive maintenance of component $i \Delta t$ time units from a tentatively planned time $t_{i}$ generated by the component's individual maintenance rule. This shift $\Delta t$ may be positive or negative (forward or backward in time). The penalty functions are derived from the individual maintenance rules in Phase 1 and usually this needs to be done only once.

Wildeman, Dekker and Smit (1996) consider two options for shifting execution times. The first option is called long-term shift, and in that case not only the current execution time $t_{i}$ is shifted, but all future maintenance of the component as well. The second option is denoted by a short-term shift, which represents the case that only the current execution time $\left(t_{i}\right)$ is shifted, and all future execution times remain unchanged. The latter is implemented by changing the interval preceding time $t_{i}$ from $x_{i}^{*}\left(=k_{i} T\right)$ to $x_{i}^{*}+\Delta t$, and by changing the interval succeeding $t_{i}$ from $x_{i}^{*}$ to $x_{i}^{*}-\Delta t$. Notice that this indeed implies that all future execution times (after $t_{i}$ ) remain the same. The short-term shift is particularly useful when the future planning of 
maintenance should not be changed. This is for example the case when we use in Phase 1 the indirect-grouping approach discussed in Section 2.1; the short-term shift leaves the coordination of maintenance as laid down by the long-term grouping as it is. Therefore, we will use the shortterm shift here.

Applying the short-term shift, the deterioration costs in the first two intervals (of length $x_{i}^{*}+$ $\Delta t$ and $x_{i}^{*}-\Delta t$, respectively) are given by $M_{i}\left(x_{i}^{*}+\Delta t\right)+M_{i}\left(x_{i}^{*}-\Delta t\right)$, whereas otherwise in each of the first two intervals $M_{i}\left(x_{i}^{*}\right)$ is paid. As all future execution times after $t_{i}$ remain unchanged, the penalty costs as a result of a shift $\Delta t$ are equal to the extra expected deterioration costs (the number of set-ups of component $i$ does not change, and the influence of other components need not be considered due to the decomposition applied), so that

$$
h_{i}(\Delta t)=M_{i}\left(x_{i}^{*}+\Delta t\right)+M_{i}\left(x_{i}^{*}-\Delta t\right)-2 M_{i}\left(x_{i}^{*}\right), \quad-x_{i}^{*}<\Delta t<x_{i}^{*} .
$$

Notice that $h_{i}(\cdot)$ is strictly convex $\left(h_{i}^{\prime \prime}(\cdot)>0\right.$ since $M_{i}(\cdot)$ is strictly convex), that $h_{i}(0)=0$, and that $h_{i}(\cdot) \geq 0$. It even holds that $h_{i}(\cdot)$ is symmetric around zero.

\section{Example}

We consider again the system with eight components which are maintained according to a standard minimal-repair model. Substituting (4) in (5), we obtain the following expressions for the penalty functions $h_{i}(\cdot)$ in that case:

$$
h_{i}(\Delta t)=c_{i}^{r}\left(\frac{x_{i}^{*}+\Delta t}{\lambda_{i}}\right)^{\beta_{i}}+c_{i}^{r}\left(\frac{x_{i}^{*}-\Delta t}{\lambda_{i}}\right)^{\beta_{i}}-2 c_{i}^{r}\left(\frac{x_{i}^{*}}{\lambda_{i}}\right)^{\beta_{i}}, \quad-x_{i}^{*}<\Delta t<x_{i}^{*} .
$$

\section{Phase 3: Tentative Planning}

Suppose the system is observed at a certain time $t$. We now consider a finite horizon with a planning of the maintenance work to be carried out during the next period. This planning is individual, and hence does not take the economic dependence between components into account. However, short-term fluctuations and opportunities can now be incorporated.

Under average operational conditions (that is, if the components are used as on average), each component $i$ is maintained $x_{i}^{*}=k_{i} T$ time units after its latest preventive maintenance. However, because of a varying use this may be at another time, depending on the utilisation rate of component $i$ since its latest execution and in the near future.

To implement a component's variable use, we define for each component a utilisation factor, and without loss of generality we assume that the average value of this factor is equal to one. This average value corresponds for example to a certain average number of running hours per day for that component. A utilisation factor of two then implies that a component is used twice as much as on average, and a factor of 0.5 corresponds to the situation where the component is used only half of the time. We assume that these factors vary for each component individually and independently.

With these utilisation factors, which are usually known only on the short term, it is easy to determine for each component $i$ its next preventive-maintenance time, which we denote by $t_{i}^{(1)}$. For example, if at the current time $t$ the latest preventive maintenance took place $y_{i}$ weeks ago, and if during that period component $i$ 's utilisation factor has been 0.5 , then at time $t$ it is as if the component has only been used for $0.5 y_{i}$ weeks. Hence, the next execution time is $k_{i} T-0.5 y_{i}$ after time $t$ (that is, $t_{i}^{(1)}=t+k_{i} T-0.5 y_{i}$ ), if indeed the utilisation factor in the near future equals one. Otherwise, if for example component $i$ 's utilisation factor in the near 
future is equal to two, then the next execution time is $\left(k_{i} T-0.5 y_{i}\right) / 2$ weeks after time $t$ (that is, $\left.t_{i}^{(1)}=t+\left(k_{i} T-0.5 y_{i}\right) / 2\right)$. If the factor attains several values since the latest execution time, the determination of $t_{i}^{(1)}$ is adapted correspondingly.

In this way we determine for each component $i$ its next preventive-maintenance time $t_{i}^{(1)}$. However, we will also consider the occurrence of the maintenance after that, the time of which is denoted by $t_{i}^{(2)}$. The execution times $t_{i}^{(1)}$ and $t_{i}^{(2)}, i=1, \ldots, n$, induce a finite planning horizon $\left[t, \max _{i} t_{i}^{(2)}\right]$, in which each component is maintained twice. The reason why we consider more than one occurrence is that otherwise the maintenance of a component $i$ may be grouped with that of another component $j$, while it could better be carried out jointly with the next occurrence of component $j$ 's maintenance. The reason why we consider two occurrences and not more, is that from our experiments it turned out that one extra occurrence for each component is sufficient. The latter can also be understood from the stability results discussed in Wildeman, Dekker and Smit (1996) with respect to the length of the planning horizon.

In this phase also opportunities can be incorporated. This is simply done by creating a dummy maintenance activity at the time the opportunity occurs. The activity has zero costs, and hence it enables other maintenance to be carried out simultaneously without paying the set-up cost $S$. If the opportunity cannot be shifted, we define its penalty functions to be infinite for every shift unequal to zero.

\section{Phase 4: Grouping Maintenance Activities}

In this phase it is allowed to shift the tentatively planned times within the planning horizon $\left[t, \max _{i} t_{i}^{(2)}\right]$ to make joint execution of maintenance possible.

A grouping structure partitions the activities in $\left[t, \max _{i} t_{i}^{(2)}\right]$ into several groups. The activities within one group are simultaneously carried out. Within one group we do not allow multiple occurrences of a component's maintenance, since this would imply that a component is maintained twice at a certain time.

In the system we are considering, preventive maintenance of component $i$ costs $s_{i}+S$, implying that joint maintenance of $m$ components yields a cost reduction of $(m-1) S$. In a group $G$ of components, the tentative execution time of component $i$ is denoted by $t_{i}$. Notice that since multiple occurrences are not allowed, $t_{i}$ is either $t_{i}^{(1)}$ or $t_{i}^{(2)}$, and no confusion is possible. The optimal execution time of group $G$ is denoted by $t_{G}^{*}$ and is found by minimising $\sum_{i \in G} h_{i}\left(t_{G}-t_{i}\right)$, which is equal to the penalty costs of maintaining the components in group $G$ at time $t_{G}$. We define the savings of group $G$ as the reduction in set-up costs minus the penalty costs, that is, $(|G|-1) S-\sum_{i \in G} h_{i}\left(t_{G}^{*}-t_{i}\right)$. A group is cost-effective if its savings are greater than or equal to zero.

Phase 4 now aims at determining an optimal grouping structure of the $2 n$ activities within

the planning horizon $\left[t, \max _{i} t_{i}^{(2)}\right]$. Such a grouping structure maximises the total savings (that is, the sum of the savings of all groups) in the planning horizon. We will apply the dynamicprogramming algorithm of Wildeman, Dekker and Smit (1996) to find an optimal grouping structure. To do so, we assume without loss of generality that the $2 n$ activities in $\left[t, \max _{i} t_{i}^{(2)}\right]$ are indexed in the order of their execution times. The algorithm terminates after $2 n$ iterations, while in each iteration $j$ a best group with last activity $j$ is found. The array entry First $[j]$ indicates the first activity of this best group. That is, if First $[j]=i$, then $\{i, \ldots, j\}$ is the best group found in iteration $j$. The total savings of the corresponding optimal grouping structure is stored in the array entry TotalSavings $[j]$. Thus, we have the following approach. 
Initialisation: TotalSavings $[0]:=0$.

Iteration 1: The best group with last activity 1 is $\{1\}$, with corresponding optimal grouping structure $\{1\}$. First $[1]:=1$. TotalSavings $[1]:=0$.

FOR $j:=2$ TO $2 n$ DO Iteration $j$ : Consider the groups with last activity $j$ in the following order: $\{j\},\{j-1, j\}, \ldots,\{1, \ldots, j\}$. Find the group for which the corresponding grouping structure covering activities $1, \ldots, j$ has largest savings. This is the group $\{i, \ldots, j\}$ for which TotalSavings $[i-1]+$ savings of $\{i, \ldots, j\}$ is maximal. First $[j]:=i$. TotalSavings $[j]:=$ TotalSavings $[i-1]+$ savings of $\{i, \ldots, j\}$.

The best grouping structure can be found by backtracking. The corresponding total savings equal TotalSavings $[2 n]$.

This algorithm can be improved by incorporating several reduction techniques, see Wildeman, Dekker and Smit (1996). This does not change the worst-case time complexity, which is equal to $\mathcal{O}\left((2 n)^{2}\right)$. However, on average an optimal grouping structure is found in less time; in the best case it requires only linear time.

\section{Phase 5: Rolling-Horizon Step}

Phase 4 provides a grouping structure for the activities in $\left[t, \max _{i} t_{i}^{(2)}\right]$. The maintenance manager can change the planning if he/she is not satisfied with it and then go back to Phase 3 ; this can be done interactively and as often as desired. Finally, the maintenance manager can carry out one or more groups of activities according to the generated grouping structure and start with Phase 3 when a planning for a new period is required.

\subsection{Comparison in Stationary Situation}

Using the above method, the dynamic-grouping approach nearly always generated in our experiments the same solutions as the stationary-grouping approach, if we assume stationarity. In the few other cases, the dynamic approach resulted in even lower costs. This can be explained as follows. Cyclic strategies such as those generated by the indirect-grouping approach are not necessarily overall optimal; sometimes it is better to use for an activity different execution intervals. For example, suppose that an optimal indirect-grouping strategy prescribes to execute an activity every six weeks, and that this implies that the activity is sometimes carried out alone. In that case, it may occasionally be better to advance or to postpone the execution by one week, and to correct this with the following maintenance interval, if thus a joint execution with other activities is possible. In our experiments we indeed encountered such examples, which the (cyclic) indirect-grouping strategy could not exploit, but which could be dealt with by our dynamic approach (where an activity need not be executed with a fixed interval). However, the extra savings obtained as a result of this were quite small. Altogether, we can conclude that the dynamic approach is consistent with the long-term approach.

Now that we have calibrated the dynamic-grouping approach, we are ready to consider the effect of short-term circumstances on the costs.

\section{The Influence of Variable Use}

Here we will obtain insight in the effect of a variable use of components on the costs according to stationary and dynamic grouping. By comparing the costs of the two approaches as a function 
of the variability in the components' use, we observe how much can be saved when short-term circumstances are not ignored but incorporated in the planning.

We assume that the utilisation factors for the components vary individually and independently; they are drawn according to a uniform distribution on the interval $[1-\delta, 1+\delta]$, where $\delta$ is the same for each component. Consequently, the parameter $\delta$ is the maximum deviation (in absolute value) from the average utilisation factor of one. By varying the value of $\delta$, we can investigate the effect of the variability in the components' use.

For reasons of simplicity, we will consider in our simulation experiments the minimal-repair model only, since in that case the cost functions can be evaluated analytically. Implementation of for example the block-replacement or inspection model, requires the numerical evaluation of the renewal function or of an integral, and this takes much time when it is done repeatedly in a simulation.

For the rate of occurrence of failures in the minimal-repair model, we take the Weibull process with scale parameter $\lambda_{i}$ and shape parameter $\beta_{i}$ for component $i$ (see (3)). The parameters $\lambda_{i}$ and $\beta_{i}$ are randomly taken from the intervals given in Table II. The intervals from which we randomly draw values for the preventive-replacement cost $s_{i}$ and the failure-repair $\operatorname{cost} c_{i}^{r}$ are also given in Table II. (Notice that the rate of occurrence of failures for the minimal-repair model is increasing, since $\beta_{i} \geq 1.5 \geq 1$.)

\section{Table II}

Data in the Simulations

\begin{tabular}{l}
\hline$\lambda_{i} \in[1,20]$ (random) \\
$\beta_{i} \in[1.5,4]$ (random) \\
$s_{i} \in[1,500]$ (random) \\
$c_{i}^{r} \in[1,250]$ (random) \\
$n=5,10,15,20$ \\
$S=10,100,500,1000$ \\
\hline
\end{tabular}

We consider four different values for the number $n$ of components: $n=5,10,15$, and 20 , and for the set-up cost $S$ we do so as well: $S=10,100,500$, and 1000. Hence, we have sixteen different combinations of $n$ and $S$, for each of which we take ten random examples as described above. All 160 examples generated in this way are solved for five different values of $\delta: \delta=0.1$, $0.3,0.5,0.7$, and 0.9. Altogether, we thus solve 800 problems.

For each problem instance, we proceed as follows. First, we solve the indirect-grouping problem (2) of Section 2.1, yielding a solution $T$ and $k=\left(k_{1}, \ldots, k_{n}\right)$. Subsequently, we randomly take for each component a utilisation factor from the interval $[1-\delta, 1+\delta]$, which is kept constant for some time; here we choose to keep the factor constant for $2 T$ time units. The precise time during which the factor is kept constant is not very important; we tried various values between one and ten, which did not have much influence on the results. For these $2 T$ time units we calculate the costs of following the long-term indirect-grouping strategy $(T, k)$, taking into account that components are used with their utilisation factor possibly differing from one. After the $2 T$ time units we repeat this process, that is, we randomly draw for each component another value of the utilisation factor from $[1-\delta, 1+\delta]$, and so on. To reduce the variance of the utilisation factor, we apply the technique of antithetic variables. That is, for each random value of a component's utilisation factor, we take a next value such that the average is equal to one. We do so with a lag of ten random drawings, that is, after ten drawings of a component's 
utilisation factor, we take the next ten values according to the technique of antithetic variables. The reason for applying a lag is that otherwise the planning of a component after two drawings is equal to that without a variable use, and this might force a coordination in the planning which is not due to randomness.

We stop the long-term planning process when two stopping criteria are simultaneously satisfied. The first criterion is that the average utilisation factor of each component has converged to the value one, which is the case after each twenty random drawings, owing to the previously described usage of antithetic variables. The second criterion is that the average costs generated by the long-term strategy have converged enough as well, which is considered to be the case when the value does not change more than a relative precision of 0.001 during $200 T$ time units.

Subsequently, we apply the rolling-horizon approach of Section 2.2 to the same problem instance. That is, we take the same simulated time and the same utilisation factors, so that a fair comparison with the long-term strategy is possible. A tentative planning is made based on the values $x_{i}^{*}=k_{i} T$, but since these values only hold for a utilisation factor equal to one, the tentative execution time of an activity is re-evaluated according to the currently known utilisation factor of the corresponding component. Together with their next occurrence, the activities induce a finite planning horizon, in which we assume that the current utilisation factors are constant; the penalty functions are evaluated according to these factors. We do so, even when the length of the finite planning horizon is longer than $2 T$ time units, in which period new utilisation factors become known. The reason for this is that in practical situations the new factors may not be known in advance. Certainly, foreknowledge would decrease the costs generated by the short-term approach. However, we do not want the results to depend on it. Based on the tentative planning, the dynamic-programming algorithm determines an optimal grouping structure, of which only the first group is implemented. If new utilisation factors are drawn before the execution time of this group, a replanning is made.

Table III summarises the results of the simulations. In this table, $g_{s t}$ denotes the average costs of the long-term indirect-grouping strategy in a stationary situation, that is, when the utilisation factors are always equal to one; $g_{l t}$ denotes the average costs when the long-term strategy is applied in case of fluctuating utilisation factors; and finally, $g_{r h}$ denotes the average costs of our rolling-horizon approach applied in that case. We define the percentual gaps of $g_{l t}$ and $g_{r h}$ over $g_{s t}$ as $\left(g_{l t}-g_{s t}\right) / g_{s t}$ and $\left(g_{r h}-g_{s t}\right) / g_{s t}$, respectively. In the table we tabulate the percentual gaps of $g_{l t}$ and $g_{r h}$ over $g_{s t}$, averaged over the 160 problem instances that we solved for each of the five different values of $\delta$.

\section{Table III}

Average Percentual Gaps of $g_{l t}$ and $g_{r h}$ over $g_{s t}$ in the 160 Problem Instances for Each of the Five Values of $\delta$

\begin{tabular}{lcccrr}
\hline & \multicolumn{1}{c}{$\delta$} & & & & \\
\cline { 2 - 6 } Strategy & 0.1 & 0.3 & 0.5 & 0.7 & \multicolumn{1}{c}{0.9} \\
\hline Long-term $(l t)$ & 0.18 & 2.11 & 6.05 & 11.98 & 20.07 \\
Dynamic $(r h)$ & -0.01 & 0.81 & 2.04 & 3.20 & 4.42 \\
\hline
\end{tabular}

From Table III we observe that the costs of following the long-term strategy increase significantly with the variability of the components' use. While for a small variability of the utilisation factor the costs are only slightly higher than $g_{s t}$, they rapidly increase for larger values of $\delta$. The costs following from our dynamic rolling-horizon approach are much less exposed to this 
effect.

In Table IV we have tabulated the minimum, average, and maximum percentual savings of applying the rolling-horizon approach compared to the long-term strategy (defined as ( $g_{l t}-$ $\left.g_{r h}\right) / g_{l t}$ ), over all 160 instances for each of the five values of $\delta$.

\section{Table IV}

Percentual Savings of $g_{r h}$ over $g_{l t}$ in the 160 Instances for Each of the Five Values of $\delta$

\begin{tabular}{lrrrrr}
\hline & \multicolumn{1}{c}{$\delta$} & & & & \\
\cline { 2 - 6 } Savings & \multicolumn{1}{c}{0.1} & 0.3 & \multicolumn{1}{c}{0.5} & \multicolumn{1}{c}{0.7} & \multicolumn{1}{c}{0.9} \\
\hline Minimum & -0.04 & 0.08 & -0.10 & 1.58 & 4.08 \\
Average & 0.19 & 1.27 & 3.76 & 7.78 & 12.88 \\
Maximum & 0.42 & 3.52 & 9.88 & 17.60 & 26.39 \\
\hline
\end{tabular}

From Table IV we observe that considerable savings are obtained when the variable use of components is taken into account. Though a utilisation factor might not fluctuate as much as 0.9 from the value one, a fluctuation of for example 0.5 is certainly not impossible, and in that case our approach yields significantly lower costs than when this short-term event is ignored. Notice that the (small) negative values in Table IV can be due to the fact that the relative precision is equal to $0.001(=0.1 \%)$.

In our simulations, we noticed an influence of the number $n$ of components; the savings averaged in Table IV seem to decrease when $n$ increases. This is primarily due to the fact that the deviation of the long-term strategy's costs $g_{l t}$ over $g_{s t}$ decreases with $n$, while the deviation of the dynamic strategy's costs $g_{r h}$ over $g_{s t}$ does not change with $n$. We do not have an explanation for this effect.

We did not identify a clear influence of the set-up cost $S$ on the results.

\section{The Influence of Opportunities}

We will investigate here how much more can be saved by dynamic grouping if not only a variable use of components is taken into account, but also the occurrence of maintenance opportunities. Opportunities can occur for different reasons. One possibility is that (unexpected) corrective maintenance of a component requires the system to be shut down, and this enables preventive maintenance of other components to be carried out simultaneously. There may also be other activities, for example the cleaning of a well in oil production, during which the system (turbines, say) can be shut down. Finally, there may be reasons outside the system, such as low-production periods, during which the shut-down costs less.

We assume that for maintenance carried out at an opportunity no set-up cost $S$ has to be paid, since this is already incurred by the opportunity itself. Hence, only the componentdependent cost $s_{i}$ has to be paid, and thus it may be worthwhile to carry out a component's maintenance activity at an opportunity when it occurs, instead of waiting until the originally planned time.

To investigate the influence of opportunities on dynamic planning, we follow our rollinghorizon approach for the 160 random examples of the previous section, with $\delta=0.5$ for the maximum variability of the components' use. Notice that although $\delta$ influences the costs that 
can be saved by our approach compared to the long-term strategy, it does not influence the extra savings that can be obtained by incorporating opportunities. Hence, the value of $\delta$ is arbitrary, but not restrictive, and it serves here only as an example. We have incorporated opportunities in the way described in Phase 3 of Section 2.2.

Although in practical situations opportunities usually occur randomly, we assume here for simplicity of implementation that opportunities occur at a deterministic interval $\eta$. Since we do not use this foreknowledge, and since the execution times of the maintenance activities are not fixed (due to the random utilisation factors), this assumption does not influence the results, and is thus justified. We choose four different values for the opportunity interval $\eta$, viz. $\eta=2$, 4, 8, and 16. As unit measure of the interval, we take the smallest maintenance interval in the indirect-grouping solution $(T, k)$ of the problem instance considered, i.e., $\min _{i}\left\{k_{i} T\right\}$. As an example, suppose that we have $\min _{i}\left\{k_{i}\right\}=2$, then if e.g. $\eta=4$, the opportunities occur each $4 \cdot 2 T=8 T$ time units in that problem instance. For each of the four values of $\eta$ we solve the 160 instances, so that in this section in total 640 problems have been solved.

Table $\mathrm{V}$ gives for $\delta=0.5$ and for the four values of $\eta$ the average percentual savings (over the 160 problem instances) of applying the rolling-horizon approach compared to the long-term strategy (defined as $\left.\left(g_{l t}-g_{r h}\right) / g_{l t}\right)$, as a function of the set-up cost $S$.

\section{Table V}

Average Percentual Savings of $g_{r h}$ over $g_{l t}$ in the 160 Instances for the Four Values of $\eta$, the Four Values of $S$, and for $\delta=0.5$

\begin{tabular}{lccccc}
\hline & $\eta$ & & & & \\
\cline { 2 - 6 } & 2 & 4 & 8 & 16 & $\infty$ \\
\hline$S=10$ & 4.30 & 4.20 & 4.16 & 4.14 & 4.13 \\
$S=100$ & 5.10 & 4.53 & 4.27 & 4.14 & 4.00 \\
$S=500$ & 8.47 & 6.13 & 4.99 & 4.46 & 3.87 \\
$S=1000$ & 9.25 & 6.06 & 4.53 & 3.81 & 3.04 \\
Average & 6.78 & 5.23 & 4.49 & 4.14 & 3.76 \\
\hline
\end{tabular}

The last column of the table $(\eta=\infty)$ corresponds to the situation where opportunities do not occur. Hence, the average savings of $3.76 \%$ for $\eta=\infty$ are equal to the average savings in Table IV for the case $\delta=0.5$ (see the previous section). A comparison of the percentual savings for $\eta=2,4,8$, and 16 , with the percentual savings for $\eta=\infty$, shows the extra savings that can be obtained when the occurrence of opportunities is taken into account, especially when the occurrence of opportunities is frequent (that is, when $\eta$ is small). Notice that for small $\eta$ the savings are increasing in $S$, while for large $\eta$ the opposite seems to be the case. However, this last effect did not occur for the other values of $\delta$, and hence we did not try to find an explanation for it; more extensive numerical experiments are necessary to decide whether this effect really occurs.

\section{Conclusions}

In this paper we showed how a long-term maintenance plan can be adapted to take short-term circumstances into account. We took a stationary- and a dynamic-planning approach from the literature and indicated how these approaches can be integrated to generate a maintenance plan 
on a rolling-horizon basis.

The numerical experiments show that dynamic planning, incorporating short-term circumstances such as a variable use of components and the occurrence of maintenance opportunities, may yield considerable cost savings compared to a long-term planning method, in which a stationary situation is assumed and hence dynamically changing information is ignored.

\section{References}

DEKKER, R. 1995. Integrating optimisation, priority setting, planning and combining of maintenance activities. European Journal of Operational Research, 82, 225-240.

Dekker, R., F.A. van der Duyn Schouten, and R.E. Wildeman. 1996. A review of multi-component maintenance models with economic dependence. Technical Report 9605/A, Econometric Institute, Erasmus University Rotterdam (accepted for publication in Zeitschrift für Operations Research).

Goyal, S.K. 1973. Determination of economic packaging frequency for items jointly replenished. Management Science, 20, 293-298.

Goyal, S.K. And A. GunaseKaran. 1992. Determining economic maintenance frequency of a transport fleet. International Journal of Systems Science, 4, 655-659.

Goyal, S.K. AND M.I. Kusy. 1985. Determining economic maintenance frequency for a family of machines. Journal of the Operational Research Society, 36, 1125-1128.

O’Connor, P.D.T. 1985. Practical Reliability Engineering. Wiley, Chichester.

Thomas, L.C. 1986. A survey of maintenance and replacement models for maintainability and reliability of multi-item systems. Reliability Engineering, 16, 297-309.

Van Eiss, M.J.G., R.M.J. Heuts, And J.P.C. Kleijnen. 1992. Analysis and comparison of two strategies for multi-item inventory systems with joint replenishment costs. European Journal of Operational Research, 59, 405-412.

Wildeman, R.E., R. Dekker, and A.C.J.M. Smit. 1996. A dynamic policy for grouping maintenance activities. Technical Report 9537/A, Econometric Institute, Erasmus University Rotterdam (to appear in the European Journal of Operational Research).

Wildeman, R.E., J.B.G. Frenk, R. Dekker, and R. van Egmond. 1995. A general approach for the coordination of maintenance frequencies. Technical Report 9539/A, Econometric Institute, Erasmus University Rotterdam (submitted for publication). 